\title{
Differential Association of Uncoupling Protein 2 Polymorphisms with Pattern Identification among Korean Stroke Patients: A Diagnostic System in Traditional Korean Medicine
}

\author{
Ji Hye Lim, ${ }^{1}$ Mi Mi Ko, ${ }^{1}$ Hoyoung Lee, ${ }^{1}$ Ho Yeon Go, ${ }^{2}$ Tae-Woong Moon, ${ }^{1}$ \\ Min Ho Cha, ${ }^{1}$ and Myeong Soo Lee ${ }^{1}$ \\ ${ }^{1}$ Medical Research Division, Korea Institute of Oriental Medicine, 1672 Yuseongdae-ro, Yuseong-gu, \\ Daejeon 305-811, Republic of Korea \\ ${ }^{2}$ Department of Korean Oriental Medicine, Semyung University, 836 Bongbang-dong, Chungju 380-960, Republic of Korea
}

Correspondence should be addressed to Min Ho Cha, mhchamin@kiom.re.kr

Received 5 March 2012; Revised 22 May 2012; Accepted 22 May 2012

Academic Editor: Ke Ren

Copyright () 2012 Ji Hye Lim et al. This is an open access article distributed under the Creative Commons Attribution License, which permits unrestricted use, distribution, and reproduction in any medium, provided the original work is properly cited.

\begin{abstract}
Uncoupling protein 2 (UCP2), a mitochondrial protein present in many organs and cell types, is known to dissipate the proton gradient formed by the electron transport chain. Its function is correlated with predictive parameters, such as obesity, diabetes, and metabolic syndromes. We analyzed the distribution of UCP2 polymorphisms in stroke patients diagnosed with one of the following four stroke subtypes based on the TKM standard pattern identification (PI): Qi-deficiency (QD), Dampness and Phlegm (D\&P), Yin-deficiency (YD), and Fire and Heat (F\&D). We studied a total of 1,786 stroke patients (397/QD, 645/D\&P, 223/YD, and 522/F\&D, 586/normal). Genotyping for the G-1957A, G-866A and A55V UCP2 polymorphisms was performed using the TaqMan. G-866A and A55V were significantly associated with the D\&P and H\&F subtypes. The frequency of subjects with the A allele of G-866A was significantly lower than the frequency of subjects with the GG type. The A55V polymorphism was also shown similar effect with G-866A in the dominant model. In contrast, no SNPs were shown to be associated with the QD or YD subtypes in this study. These results showed that the G-866A and A55V UCP2 polymorphisms may be genetic factors for specific PI types among Korean stroke patients.
\end{abstract}

\section{Introduction}

The uncoupling proteins (UCPs) are a protein family comprised of mitochondrial proteins located at the inner membrane of mitochondria [1]. They function in the dissipation of proton gradients formed by the electron transport system and play roles in the homeostasis of body temperature by thermogenesis and in decreasing ROS production $[2,3]$. The UCP family is composed of five subtypes: UCP1, UCP2, UCP3, UCP4, and UCP5. UCP1 and UCP3, which both function in thermogenesis, have tissue-specific expression patterns. The major sites of UCP1 and UCP3 expression are brown adipose tissue and muscle, respectively $[1,4]$. Unlike UCP1 and UCP3, UCP2 is widely expressed in all tissues and reduces reactive oxygen species (ROS) production [3]. ROS have important roles in cell signaling and homeostasis
$[5,6]$, but increase of ROS level by environmental stress and cellular metabolism causes abnormal inflammatory responses, atherosclerosis and cardiovascular disease [7-11], and UCP2 is involved in the preservation of ROS homeostasis and is associated with many cardiovascular diseases.

The UCP2 gene is located at chromosome 11q13, and many studies have reported the association of the G-866A and $\mathrm{A} 55 \mathrm{~V}$ polymorphisms of UCP2 with various diseases, such as obesity, diabetes, and metabolic syndromes [2, $12-$ 15]. For example, A55V was significantly associated with obesity in men of Japanese. The frequency of subjects with the AV or VV types in the obese group was $67.4 \%$, which was significantly lower than the frequency observed in the nonobese group (78.1\%) [14]. Another report showed that G-866A was associated with a reduction in the prevalence of type 1 diabetes in Germany [15]. These data suggest that 
polymorphisms of UCP2 are associated with obesity and its related metabolic syndromes and diseases.

In traditional Korean medicine (TKM), subjects with disease are diagnosed according to pattern identification (PI). PI is a comprehensive system for the diagnosis of disease according to the subject's signs and symptoms. The signs and symptoms reflect a personal state; thus, PI is a diagnostic system for the cause, nature, and location of the illness, the patient's physical condition, and the patient's treatment [16-21]. TKM classifies stroke into four sub-PI groups: Qi-deficiency (QD), Dampness and Phlegm (D\&P), Yindeficiency (YD), and Fire and Heat (F\&D) [22]. Among the PI subtypes defined as follows according to Min et al. [23], the D\&P is characterized by its impediment to Qi movement, turbidity, heaviness, stickiness, and downward flowing properties and shows an eminent tendency toward obesity and increase of serum lipids. The H\&F accounts for pathogenic fire characterized by intense heat that is apt to injure fluid and to consume Qi. The YD indicates pathologic change marked by deficiency of Yin and being known to highly contribute to emaciation, rarely to obesity. The QD is a deficiency of qi that leads to decreased visceral function and lowered body resistance.

PI is affected by hereditary factor as well as environmental factors. Some reports showed relationships between genetic polymorphisms and PI in Chinese, Korean, and Japanese populations [24-27]. Finding genes related with stroke-PI, we previously reported that NPY and PON1 polymorphism wass associated with the D\&P [24, 28]. Because UCP2 is involved in cellular metabolism and thermogenesis, and some SNPs in UCP2 were known to be associated with obese/overweight and serum lipid, we hypothesized that SNPs, changing UCP2 expression or amino acid, might be related with PI subtypes including the F\&H related with thermogenesis and the D\&P, YD related with metabolism imbalance. In this study, we investigated the G-1987A, G866A, and A55V UCP2 polymorphisms in 586 normal subjects and 1,786 stroke patients diagnosed by two expert TKM doctors using PI, and we thereby elucidated the association of UCP2 polymorphisms with PI.

\section{Subjects and Methods}

2.1. Subjects. We examined 1,786 subjects diagnosed with stroke and 586 normal subjects without stroke. The stroke patients were admitted into the twelve oriental medical hospitals participating in this study; these hospitals are located throughout South Korea to minimize regional differences: KyungHee Oriental Medical Center (Seoul), KyungHee East-West Neo Medical Center (Seoul), DongGuk International Hospital (Kyunggi-do), Kyungwon Oriental Medical Hospitals (Seoul and Inchon), DongSeo Oriental Medical Hospital (Seoul), DaeJeon Oriental Medical Hospital (Daejeon), DongSin Oriental Medical Hospital (Gwangju and Jeollanam-do), Wonkwang Oriental Medical Hospital (Jeollabuk-do), Woo Suk Oriental Medical Hospital (Jeollabuk-do), and SangJi Oriental Medical Hospital (Gangwon-do). Some of the included subjects overlapped with our previous study [24]. The stroke diagnosis was confirmed by brain computer tomography (CT), magnetic resonance imaging (MRI), or magnetic resonance angiography (MRA), and the clinical data from the subjects were collected after obtaining written informed consent. The normal subjects were recruited from DaeJeon Oriental Medical Hospital and Wonkwang Oriental Medical Hospital, and stroke status was confirmed by MRI. This study was performed after receiving approval by the Institutional Review Boards of the Korean Institute of Oriental Medicine and by each of the Oriental Medical Hospitals.

2.2. PI Diagnosis of Stroke Patients. The PI diagnosis of subjects was performed as described in the report published by Lee et al. [22]. Briefly, the signs and symptoms presented by the patients were classified using the "Stroke PI case report form" [16]. The PI diagnosis of each patient was determined by two expert TKM doctors, and subjects receiving differing diagnoses from the two doctors were excluded. The number of patients in each PI group was as follows: 397 in the QD group, 645 in the D\&P group, 223 in the YD group, and 522 in the H\&D group.

2.3. Genomic DNA Preparation and Genotyping. Genomic DNA from each subject was prepared from blood using the GeneAll genomic DNA extraction kit (GeneAll, Seoul, Korea). The UCP2 single-nucleotide polymorphisms (SNPs) analyzed in this study are listed in Table 2. The genotyping of all analyzed SNPs was performed by Macrogen Inc. (Seoul, Korea) using the polymerization chain reaction (PCR) with TaqMan probes. To confirm the accuracy of the genotype analysis, five percent of our subjects were randomly selected, and their SNPs were genotyped by the sequencing method using the ABI 3700 sequencer (ABI Inc. Carlsbad, CA USA). The SNP-genotyping agreement between the two methods was $97.95 \%$, which represents high accuracy. The concordance of UCP2 SNPs in normal with Hardy-Weinberg equilibrium (HWE) was tested using HapAnalyzer software [29].

2.4. Statistical Analysis. The statistical analysis of our data was performed with SAS software version 9.1.3 (SAS Institute Inc., Cary, NC USA). Differences in continuous variables were determined by nonparametric (Wilcoxon rank-sum test) tests. Categorical variables were compared with a chi-squared test or Fisher's exact test. A multiple logistic regression adjusted for age, sex, smoking status, and drinking status was performed to estimate the association of SNPs with the normal group versus each of the PI groups, and odds ratios (ORs) with 95\% confidence intervals (95\% CI) were obtained. We performed a statistical analysis using a general linear model adjusted for age, sex, and smoking status to investigate whether the PIs are associated with the serum parameters of the patient subjects. The statistical significance was set at $P<0.05$. 


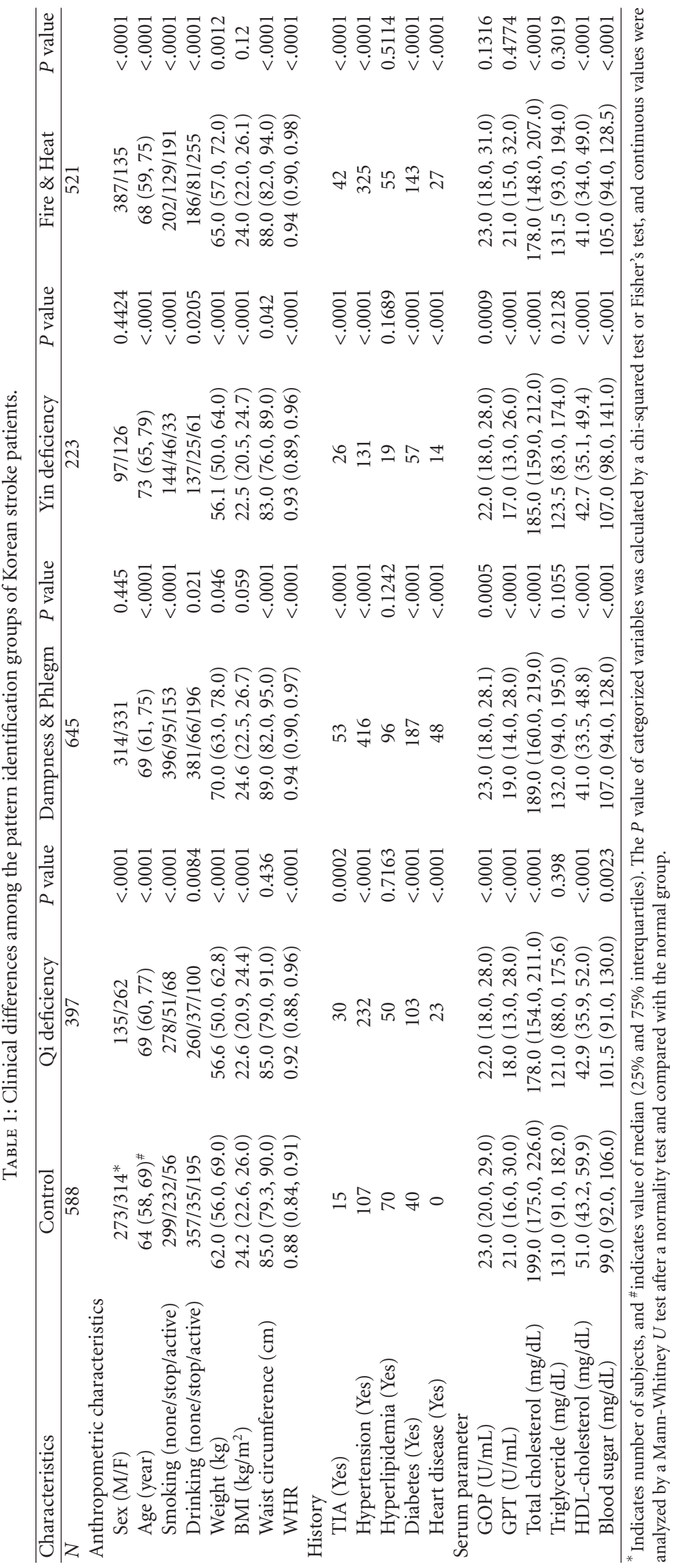


TABLE 2: List of the SNPs analyzed in this study.

\begin{tabular}{lcccccrr}
\hline SNP & rs no. & Position & $\begin{array}{c}\text { Location relative } \\
\text { to transcription } \\
\text { start site }\end{array}$ & $\begin{array}{c}\text { Nucleotide } \\
\text { (amino acid) } \\
\text { change }\end{array}$ & $\begin{array}{c}\text { Location relative } \\
\text { to p-terminus of } \\
\text { chromosome }\end{array}$ & MAF & HWEp \\
\hline G-1957A & rs649446 & Promoter & -1957 & G/A & 73695845 & 0.3147 \\
G-866A & rs659366 & Promoter & -866 & G/A & 73694754 & 0.138 \\
A55V & rs660339 & exon 2 & 4787 & G/A (A/V) & 73689104 & 0.4871 & 0.051 \\
\hline
\end{tabular}

\section{Results}

Table 1 shows the clinical differences between each of the PI groups and the normal group. The history of disease and the serum parameters of each group displayed a pattern similar to that of our previous reports [24, 25]. However, obese indices, such as BMI and waist circumference, showed a significantly different pattern in the PI groups compared with the normal group. The median BMIs of the QD and YD groups were $22.6 \mathrm{~kg} / \mathrm{m}^{2}$ and $22.5 \mathrm{~kg} / \mathrm{m}^{2}$, respectively, which were significantly lower than the $24.2 \mathrm{~kg} / \mathrm{m}^{2}$ BMI of the normal subjects $(P<0.001)$. In contrast, the BMIs of the $\mathrm{D} \& \mathrm{P}$ and $\mathrm{H} \& \mathrm{~F}$ groups were not different compared to the normal group $(P=0.056$ and $P=0.120$, resp.). Waist circumference showed the opposite pattern; it was significantly increased in the $\mathrm{D} \& \mathrm{P}$ and $\mathrm{H} \& \mathrm{~F}$ groups, but not in the QD and YD groups, compared to the normal group. Differences in the body characteristics and serum lipid parameters among the PI groups were shown in supplemental available online at doi:10.1155/2012/532078. The mean of obese indices such as BMI, waist circumference and WHR were significantly higher in the D\&P and H\&F groups than YD and Qi groups, and the level of triglycerides in the D\&P group was also higher in the D\&P group than that of the other three groups. The location of three SNPs within the UCP2 gene is shown in Figure 1(a), and their linkage distribution among three SNPs in Figure 1(b).

The characteristics of the SNPs investigated in this study are listed in Table 2. Two of the SNPs are located at the -1957 and -866 positions relative to the transcriptional start site. The other SNP is situated at amino acid 55 in UCP2 exon 4 , which causes the change of an alanine to a valine. All of the alleles were in Hardy-Weinberg equilibrium $(P>$ 0.05) in the normal group, according to the recommended International HapMap Project guidelines. An analysis of the linkage distribution among the three SNPs indicated that G866A and A55V were tightly linked. The $D^{\prime}$ and $r^{2}$ values were 1.00 and 0.97 , respectively, which are similar to those reported in a previous report by Cha et al. [30].

Table 3 shows the SNP distribution in each PI group compared to the normal group. G-866A was significantly associated with D\&P and H\&F. The ratio of subjects with the A allele was $69.58 \%$ in the D\&P group and $71.92 \%$ in the H\&F group, both of which were significantly lower than the $76.66 \%$ ratio of the normal group, after adjusting for sex, age, smoking, and drinking $(\mathrm{OR}=0.668$ (0.5010.890), $P=0.006$; OR $=0.647$ (0.472-0.886), $P=0.007$, resp.). The A55V SNP, which is tightly linked with G-866A, showed similar pattern of G-866A in the dominant model.

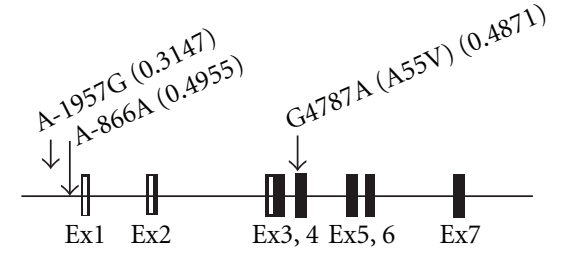

(a)

\begin{tabular}{|c|c|c|c|c|}
\hline & & \multicolumn{3}{|c|}{$\left|D^{\prime}\right|$} \\
\hline & & G-1957A & G-866A & A55V \\
\hline \multirow{3}{*}{$r^{2}$} & G-1957A & & 0.979 & 0.972 \\
\hline & G-866A & 0.453 & & 0.989 \\
\hline & A55V & 0.455 & 0.96 & \\
\hline
\end{tabular}

(b)

FIgure 1: (a) Map of the SNPs located in the UCP2 gene. The values in parentheses show the minor allele frequency. The open and closed boxes in the exons indicate the untranslated and translated regions, respectively. (b) Linkage distribution among three SNPs.

The frequency of A allele of $\mathrm{A} 55 \mathrm{~V}$ is $68.97 \%$ in the $\mathrm{D} \& \mathrm{P}$ group and $70.27 \%$ in the $\mathrm{H} \& \mathrm{~F}$ group, which are significantly lower than the $75.6 \%$ ratio of the normal group $(P=0.010$ in $\mathrm{D} \& \mathrm{P}, P=0.007$ in $\mathrm{H} \& \mathrm{~F})$. In contrast, no SNPs were associated with QD or YD in this study.

To confirm which factor was affected by G-866A or $\mathrm{A} 55 \mathrm{~V}$, we compared serum lipid parameters according to genotype in normal subjects in Table 4. A55V was associated with serum triglyceride. The mean serum triglyceride of the subjects with the AV and VV type at the 55 position of the UCP2 gene was $142.6 \mathrm{mg} / \mathrm{dL}$ and $148.82 \mathrm{mg} / \mathrm{dL}$, respectively. These means were significantly lower than the mean serum triglyceride $(158.58 \mathrm{mg} / \mathrm{dL})$ of the subjects with the AA type in the dominant mode $(P=0.0471)$.

\section{Discussion}

Stroke is the second most common cause of death in Korea; 52 women and 50.3 men per one hundred thousand people died because of stroke in 2009 [31]. Therefore, many efforts to prevent the occurrence of stroke are necessary. Additionally, the rapid and appropriate treatment of stroke is also important to remove or reduce the aftereffects of a stroke. Recently, personalized medicine has been emphasized; in this model, instead of the uniform treatment of 


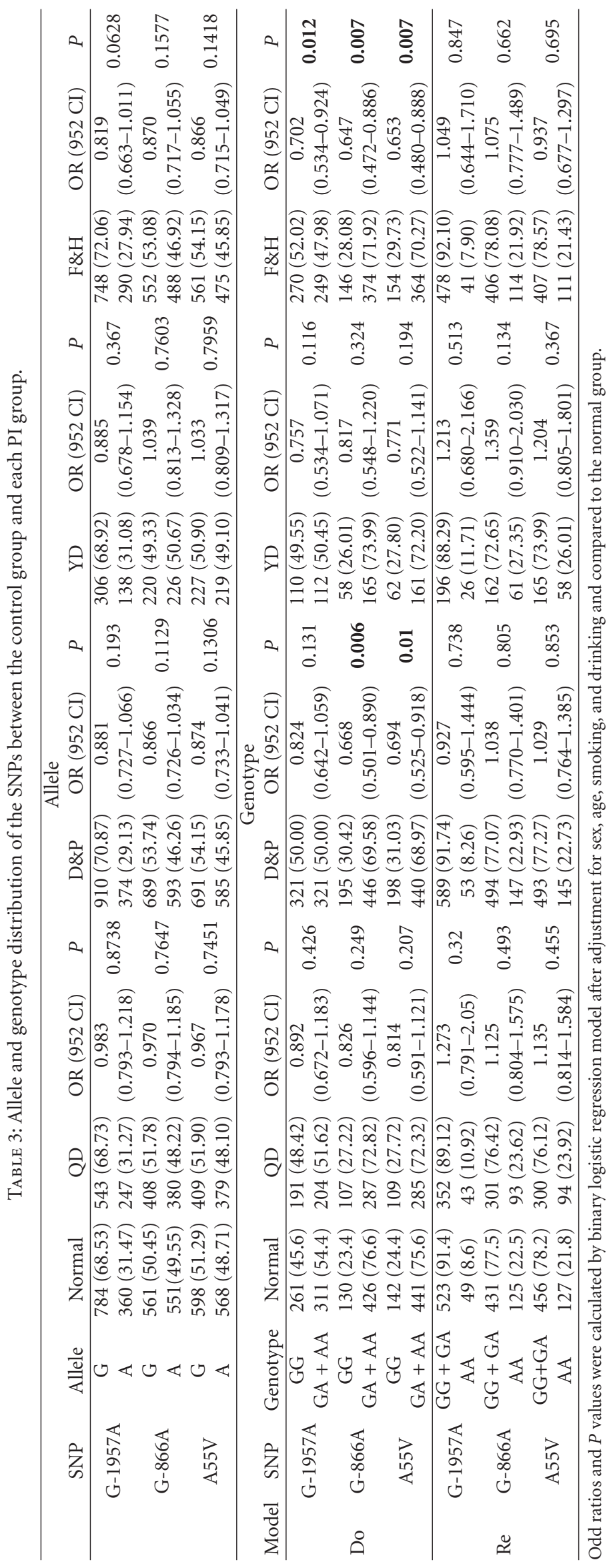




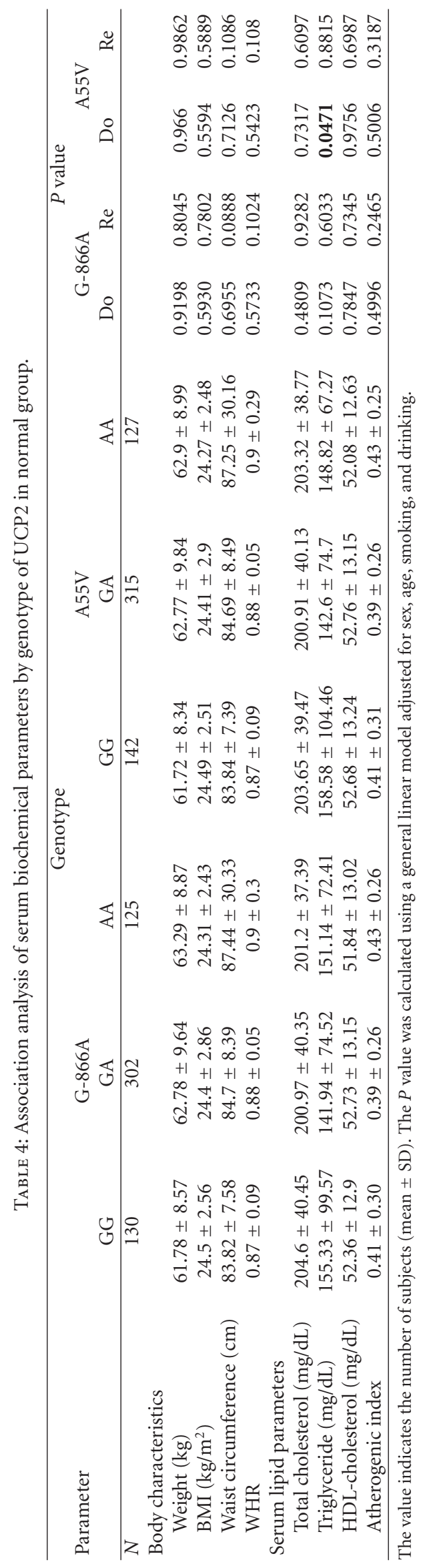


disease, patients receive different treatments according to the patient's individual condition, which might increase the positive effects of treatment and reduce the adverse effects [32-34]. In TKM, patients with the same disease are given different treatments depending on the patient's condition. PI, a basic system for diagnosis in TKM, entails a systematic analysis of the patient's physical condition. Based on PI, TKM doctors determine the cause, nature, and treatment of the illness [16]. Because of these shared aspects, treatments based on PI might approximate personalized medicine, and some recent studies have been undertaken to identify genetic variation associations with PI $[24,27]$.

In TKM, stroke is classified into four standard subtypes according to PI: Qi-deficiency, Dampness and phlegm, Yindeficiency, and Fire and Heat [22]. D\&P is correlated with obesity and obesity-related metabolic syndromes [23, 24]. For example, Min et al. reported that total cholesterol, BMI, and waist circumference were significantly increased in D\&P pattern patients [23], and this finding is similar to our results (Table 1). We also found that the clinical characteristics of the H\&F pattern were similar to the D\&P pattern.

In the current study, we investigated the association of UCP2 polymorphisms with PI. UCP2 is a mitochondrial protein and is known to dissipate the proton gradient formed by electron transport and decrease ROS production [2]. The G-866A polymorphism located at the promoter region and the $\mathrm{A} 55 \mathrm{~V}$ polymorphism in exon 4 of the UCP2 gene were associated with thermogenesis and energy consumption in lipids metabolism, resulting in weight loss and low serum lipid level in Korean population studies $[34,35]$. In this study, the G-866A SNP had a negative effect on the D\&P and $\mathrm{H} \& \mathrm{~F}$ patterns. $\mathrm{A} 55 \mathrm{~V}$, which is a nonsynonymous SNP and is linked with G-866A, also showed a negative association with the D\&P and H\&F patterns (Table 3). Obesity indices, such as BMI, WHR, and serum lipids, showed an increase in the D\&P and H\&F patterns compared with the QD and YD patterns (Table 4). These results might suggest that the D\&P and H\&F patterns are related to obesity or that obese subjects have a lower ratio of GA and AA genotypes of the G-866A SNP. This result matched that of a previous study reported by Jun et al. [36]. In the report, G-866A and A55V were associated with a decrease of BMI and waist circumference in Korean children [36]. Other reports studying other populations also showed negative effects of G-866A on obesity $[12,14]$. However, our result was in contrast to other studies reported by Wang et al. and Martinez-Hervas et al. [37, 38], who showed an increased susceptibility to obesity for two SNPs in Taiwanese and southern European populations.

In TKM, the PI is classified by position, internal or external etiology, or organ. The D\&P and H\&F patterns are classified by etiology. D\&P is a combination of phlegm and internal dampness that causes disease. D\&P produces anorexia, dyspepsia, dizziness, headache, heaviness, and other maladies. H\&F is any pattern or syndrome of heat and fire, either contracted externally or engendered internally. $\mathrm{H} \& \mathrm{~F}$ produces hot flashes, mouth or tongue dryness, ophthalmoxerosis, and other ailments of the body [39, 40]. In TKM, D\&P and F\&H could be explained as causation in the pathogenesis. When damp is congested for a long time and phlegm arises from the body, damp and phlegm are mixed up each other. The longer damp and phlegm stay in the body, the more heat they generate. What is worse, D\&P are combined with the heat that they generate and start to produce more complicated symptoms attributed to the mixture of damp, phlegm, and heat [41]. In addition, the F\&H seems to induce so excessive heat generation and high-energy consumption that it can result in the obesity by increasing the dietary intake to supplement the energy dearth.

In this study, we showed an association of UCP2 polymorphisms with PI in Korean stroke patients. UCP2 polymorphisms, far involved in metabolism and thermogenesis, were significantly distributed to $\mathrm{D} \& \mathrm{P}$ and $\mathrm{F} \& \mathrm{H}$, indicating that the $\mathrm{F} \& \mathrm{H}$ along with the $\mathrm{D} \& \mathrm{P}$ was another obese factor. However, this study had some limitations that prevent generalizing these results. The first limitation is the sample size disparity between the test and control group that may have impacted finding. Secondly, the PI was limited to stroke diagnoses. The lack of evidence on the accuracy of PI forced us to perform an observational study, not allowing a randomized controlled trial. Thus, further studies with greater balanced sample size that focus on other diseases will be necessary to confirm the scientific basis of the relationship between genetic variations and PI.

\section{Acknowledgment}

This research was supported by a Grant from the Korea Inst. of Oriental Medicine (K12130).

\section{References}

[1] S. Klaus, L. Casteilla, F. Bouillaud, and D. Ricquier, "The uncoupling protein UCP: a membraneous mitochondrial ion carrier exclusively expressed in brown adipose tissue," International Journal of Biochemistry, vol. 23, no. 9, pp. 791801, 1991.

[2] C. Fleury, M. Neverova, S. Collins et al., "Uncoupling protein2: a novel gene linked to obesity and hyperinsulinemia," Nature Genetics, vol. 15, no. 3, pp. 269-272, 1997.

[3] G. Mattiasson and P. G. Sullivan, "The emerging functions of UCP2 in health, disease, and therapeutics," Antioxidants and Redox Signaling, vol. 8, no. 1-2, pp. 1-38, 2006.

[4] S. B. Pedersen, S. Lund, E. S. Buhl, and B. Richelsen, "Insulin and contraction directly stimulate UCP2 and UCP3 mRNA expression in rat skeletal muscle in vitro," Biochemical and Biophysical Research Communications, vol. 283, no. 1, pp. 19$25,2001$.

[5] C. S. Zong, J. L. K. Chan, S. K. Yang, and L. H. Wang, "Mutations of Ros differentially effecting signal transduction pathways leading to cell growth Versus transformation," Journal of Biological Chemistry, vol. 272, no. 3, pp. 1500-1506, 1997.

[6] K. Chen, S. R. Thomas, and J. F. Keaney, "Beyond LDL oxidation: ROS in vascular signal transduction," Free Radical Biology and Medicine, vol. 35, no. 2, pp. 117-132, 2003.

[7] K. K. Griendling and G. A. FitzGerald, "Oxidative stress and cardiovascular injury part I: basic mechanisms and in vivo monitoring of ROS," Circulation, vol. 108, no. 16, pp. 1912 1916, 2003. 
[8] C. Zhang, J. Liu, H. Pan, X. Yang, and K. Bian, "Mitochondrial dysfunction induced by excessive ROS/RNS-metabolic cardiovascular disease and traditional Chinese medicines intervention," Zhongguo Zhong Yao Za Zhi, vol. 36, pp. 24232428, 2011.

[9] J. C. Kim, Y. H. Lee, M.-K. Yu et al., "Anti-inflammatory mechanism of PPAR gamma on LPS-induced pulp cells: role of the ROS removal activity," Archives of Oral Biology, vol. 57, no. 4, pp. 392-400, 2011.

[10] S. Zhao, L. Zhang, G. Lian et al., "Sildenafil attenuates LPSinduced pro-inflammatory responses through down-regulation of intracellular ROS-related MAPK/NF- $\kappa \mathrm{B}$ signaling pathways in N9 microglia," International Immunopharmacology, vol. 11, no. 4, pp. 468-474, 2011.

[11] A. Stirban, "The role of AGEs and ROS in atherosclerosis," Herz, vol. 35, no. 3, pp. 170-180, 2010.

[12] H. Esterbauer, C. Schneitler, H. Oberkofler et al., "A common polymorphism in the promoter of UCP2 is associated with decreased risk of obesity in middle-aged humans," Nature Genetics, vol. 28, no. 2, pp. 178-183, 2001.

[13] C. B. Chan, "Endogenous regulation of insulin secretion by UCP2," Clinical Laboratory, vol. 48, no. 11-12, pp. 599-604, 2002.

[14] K. Kosuge, M. Soma, T. Nakayama et al., "Human uncoupling protein 2 and 3 genes are associated with obesity in Japanese," Endocrine, vol. 34, no. 1-3, pp. 87-95, 2008.

[15] M. Sakaue, M. Kawabe, and H. Taniguchi, "Polymorphisms of uncoupling protein-1 gene in type 2 diabetes," Japanese Journal of Clinical Medicine, vol. 63, pp. 202-206, 2005.

[16] H. J. Kim, H. S. Bae, S. U. Park, S. K. Moon, J. M. Park, and W. S. Jung, "Clinical approach to the standardization of oriental medical diagnostic pattern identification in stroke patients," Evidence-Based Complementary and Alternative Medicine, vol. 2011, Article ID 768492, 7 pages, 2011.

[17] WHO, WHO International Standard Terminologies on Traditional Medicine in the Western Pacific Region, WHO Western Pacific Regional Office, 2007.

[18] B. K. Kang, T. Y. Park, T. W. Moon et al., "Reliability and validity of a Pattern Identification Questionnaira for Koran stroke patients," BMC Complementary and Alternative Medicine, vol. 12 , article 55, 2012.

[19] J. A. Lee, T. Y. Park, J. Lee et al., "Developing indicators of pattern identification in patients with stroke using traditional Korean medicine," BMC Research Notes, vol. 5, article 136, 2012.

[20] T. Y. Park, J. A. Lee, M. H. Cha et al., "The fundamental study for the standardization and objectification of pattern identification in traditional Korean medicine for stroke (SOPIStroke): an overview of phase I," European Journal of Integrative Medicine, vol. 4, pp. e125-e131, 2012.

[21] B. K. Kang, T. W. Moon, J. A. Lee, T.-Y. Park, M. M. Ko, and M. S. Lee, "The fundamental study for the standardization and objectification od pattern identification in traditional Korean medicine for stroke (SOPI-stroke): development and interobserver agreement of the Korean standard pattern identification for stroke (K-SPI-Stroke) tool," European Journal of Integrative Medicine, vol. 4, pp. e133-e139, 2012.

[22] J. A. Lee, J. S. Lee, M. M. Ko et al., "Report on the Korean standard pattern identifications for stroke-III," Korean Journal of Oriental Internal Medicine, vol. 32, no. 1, pp. 232-242, 2011.

[23] I. K. Min, C. H. Kim, J. W. Hwang et al., "The relation of Dampness-Phlegm and metabolic syndrome in acute stroke patients," Journal of Korean Oriental Medicine, vol. 30, no. 1, pp. 109-119, 2009.
[24] J. H. Lim, M. M. Ko, J. S. Lee, O. S. Bang, and M. H. Cha, "Genetic association of SNPs located at PON1 gene with Dampness and phelgm pattern identification among Korea stroke patients," Korean Journal of Oriental Internal Medicine, vol. 31, no. 4, pp. 752-762, 2010.

[25] T. Ouyang, J. N. Song, Y. Miao et al., "Study on relationship between polymorphism of apolipoprotein E gene and syndromes of phlegm and blood stasis in patients with coronary heart disease," Journal of Chinese Integrative Medicine, vol. 3, no. 6, pp. 438-442, 2005.

[26] S. An, E. Li, and X. Tong, "Study on relationship between estrogen receptor gene polymorphism and syndrome differentiation typing of female postmenopausal osteoporosis in Traditional Chinese medicine," Chinese Journal of Integrated Traditional and Western Medicine, vol. 20, no. 12, pp. 907-910, 2000.

[27] S. Chen, F. Lv, J. Gao et al., "HLA class II polymorphisms associated with the physiologic characteristics defined by traditional Chinese medicine: linking modern genetics with an ancient medicine," Journal of Alternative and Complementary Medicine, vol. 13, no. 2, pp. 231-239, 2007.

[28] M. M. Ko, B. K. Kang, J. H. Lim, M. S. Lee, M. H. Cha et al., "Genetic association of NPY gene polymorphisms with dampness-phlegm pattern in Korean stroke patients," Evidence Based Complement Alternative Medicine, vol. 2012, Article ID 109796, 7 pages, 2012.

[29] N. S. Kim, S. M. Oh, M. M. Ko, M. H. Cha, B. K. Kang, and O. S. Bang, "Association of the C-399T promoter polymorphism of neuropeptide Y with susceptibility to ischemic stroke," Clinical Biochemistry, vol. 42, no. 16-17, pp. 1699-1704, 2009.

[30] M. H. Cha, I. C. Kim, K. S. Kim, B. K. Kang, S. M. Choi, and Y. Yoon, "Association of UCP2 and UCP3 gene polymorphisms with serum high-density lipoprotein cholesterol among Korean women," Metabolism: Clinical and Experimental, vol. 56, no. 6, pp. 806-813, 2007.

[31] Annual report on the cause of death statistics, Statistics Korea.

[32] I. Kirstein-Grossman, J. S. Beckmann, D. Lancet, and A. Miller, "Pharmacogenetic development of personalized medicine: multiple sclerosis treatment as a model," Drug News and Perspectives, vol. 15, no. 9, pp. 558-567, 2002.

[33] A. H. Trainer, B. Meiser, K. Watts, G. Mitchell, K. Tucker, and M. Friedlander, "Moving toward personalized medicine: treatment-focused genetic testing of women newly diagnosed with ovarian cancer," International Journal of Gynecological Cancer, vol. 20, no. 5, pp. 704-716, 2010.

[34] K. E. Morrison, "Whole-genome sequencing informs treatment: personalized medicine takes another step forward," Clinical Chemistry, vol. 57, no. 12, pp. 1638-1640, 2011.

[35] Y. Yoon, B. L. Park, M. H. Cha et al., "Effects of genetic polymorphisms of UCP2 and UCP3 on very low calorie diet-induced body fat reduction in Korean female subjects," Biochemical and Biophysical Research Communications, vol. 359, no. 3, pp. 451-456, 2007.

[36] H. S. Jun, I. K. Kim, H. J. Lee et al., "Effects of UCP2 and UCP3 variants on the manifestation of overweight in Korean children," Obesity, vol. 17, no. 2, pp. 355-362, 2009.

[37] H. Wang, W. S. Chu, T. Lu, S. J. Hasstedt, P. A. Kern, and S. C. Elbein, "Uncoupling protein-2 polymorphisms in type 2 diabetes, obesity, and insulin secretion," American Journal of Physiology, vol. 286, no. 1, pp. E1-E7, 2004.

[38] S. Martines-Hervas, M. L. Mansego, G. de Marco et al., "Polymorphisms of the UCP2 gene are associated with body fat distribution and risk of abdominal obesity in Spanish 
population," European Journal of Clinical Investigation, vol. 42, no. 1, pp. 171-178, 2011.

[39] WHO, WHO International Standard Terminologies on Traditional Medicine in the Western Pacific Region, World Health Organization, 2007.

[40] M. M. Ko, J. A. Lee, B.-K. Kang et al., "Interobserver reliability of tongue diagnosis using traditional Korean Medicine for stroke patients," Evidence-Based Complementary and Alternative Medicine, vol. 2012, Article ID 209345, 6 pages, 2012.

[41] C. K. Park, "Etiology and pathology of traditional Korean medicine," Traditional Korean Medicine Research Institute, pp. 1-610, 1992. 


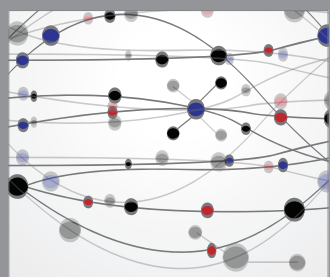

The Scientific World Journal
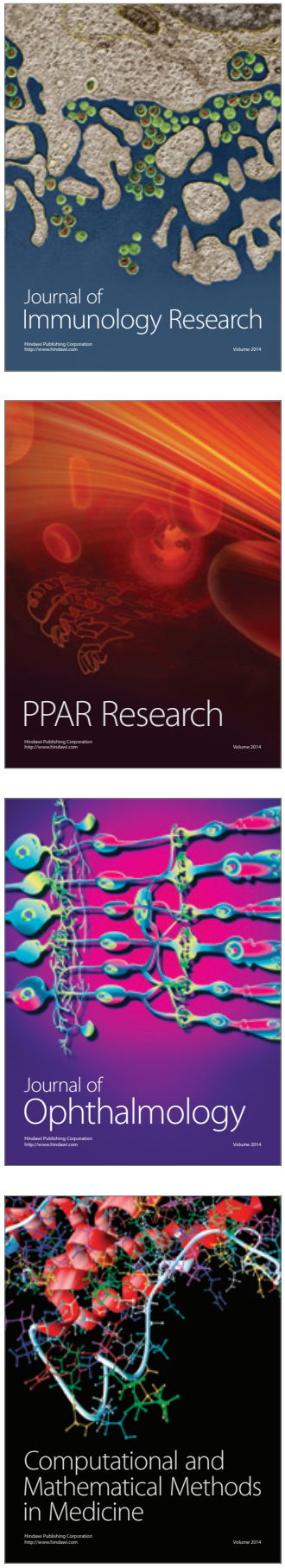

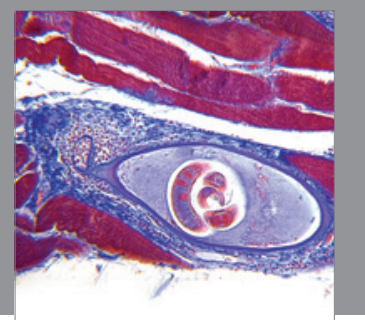

Gastroenterology

Research and Practice
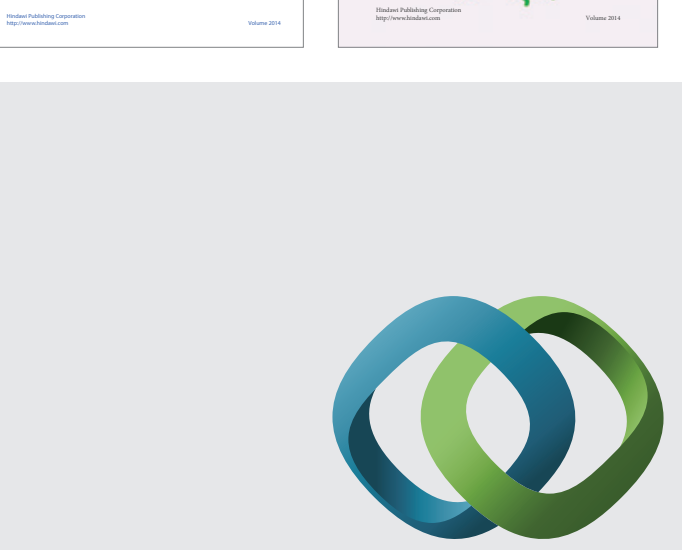

\section{Hindawi}

Submit your manuscripts at

http://www.hindawi.com
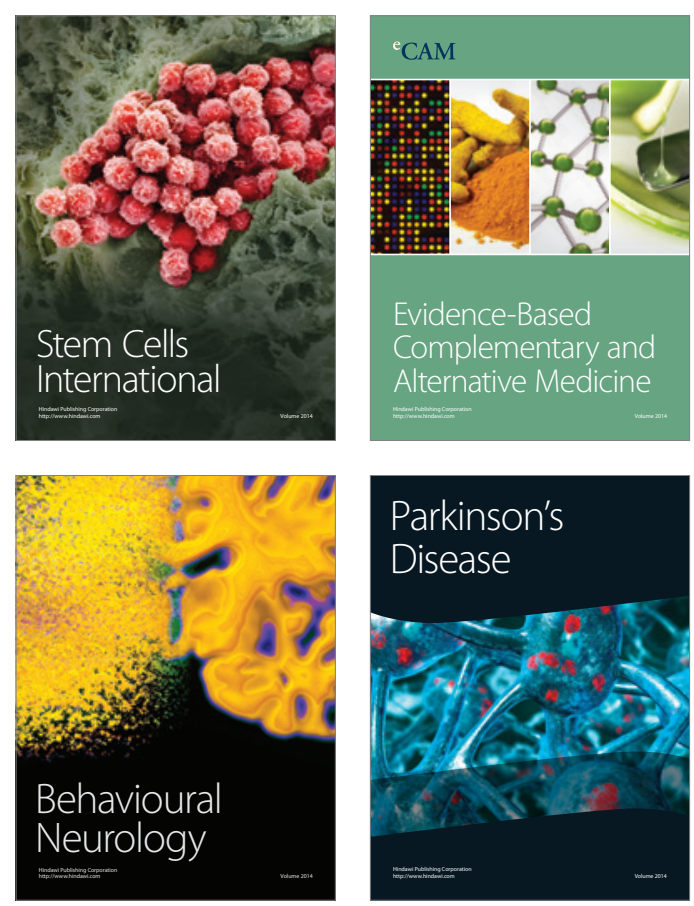

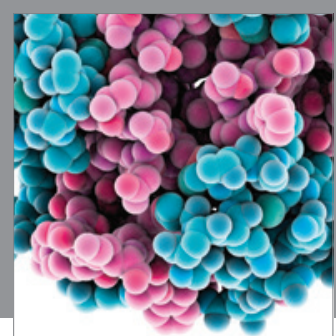

Journal of
Diabetes Research

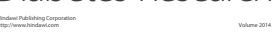

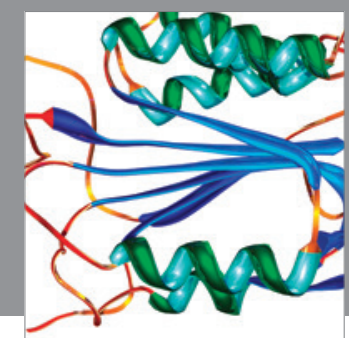

Disease Markers
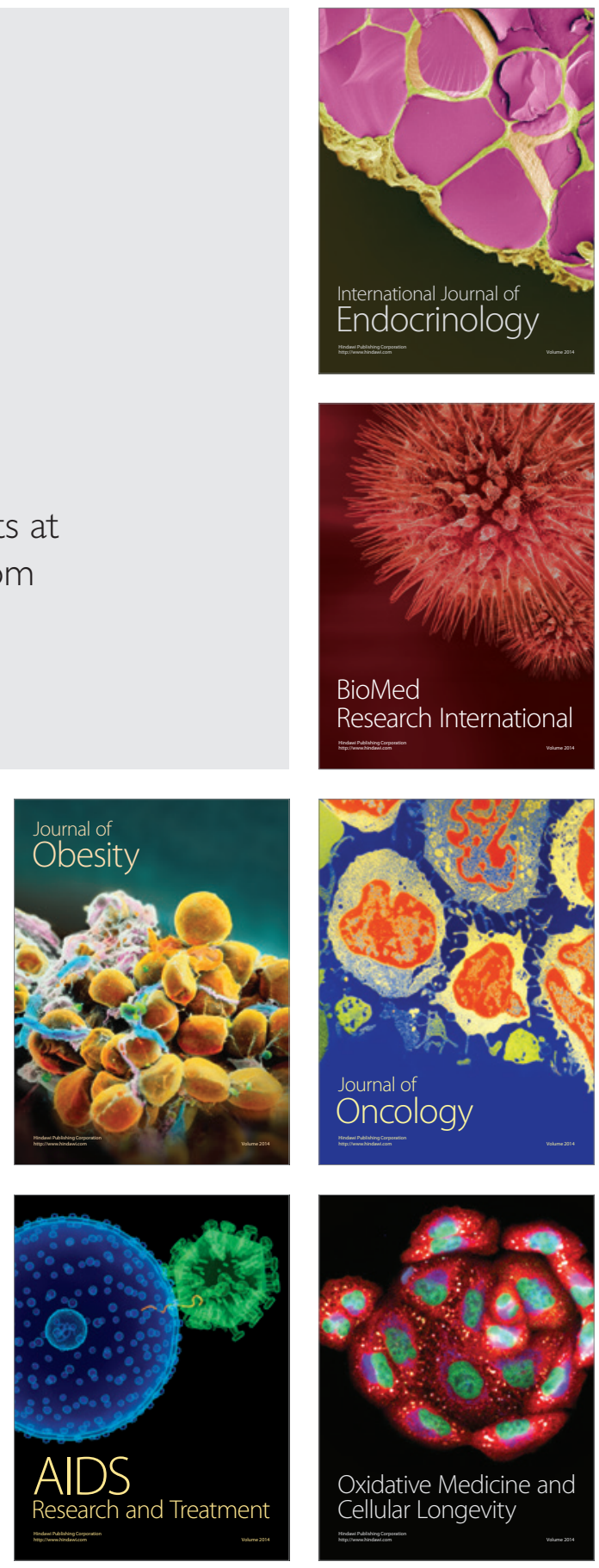